\title{
Thermo-elastic analysis of a cracked substrate bonded to a coating using the hyperbolic heat conduction theory
}

\author{
Zengtao Chen ${ }^{1, *}$, Keqiang $\mathrm{Hu}^{1}$ \\ ${ }^{1}$ Department of Mechanical Engineering, University of New Brunswick, Fredericton NB E3B 5A3, Canada \\ * Corresponding author: ztchen@unb.ca
}

\begin{abstract}
In this paper, the dynamic thermal stresses around a crack in a substrate bonded to a coating are obtained using the hyperbolic heat conduction theory. Fourier and Laplace transforms are applied and the hyperbolic heat conduction and thermo-elastic crack problems are reduced to solving singular integral equations. The crack kinking phenomenon under thermal loading is investigated by applying the criterion of maximum hoop stress. Numerical results show that the hyperbolic heat conduction parameters, the material properties and the geometric size of the composite have significant influence on the dynamic stress field. It seems that high temperature loading on the surface may lead to crack kinking away from the surface and low temperature loading may cause crack kinking toward the coating. Moreover, the hyperbolic heat conduction theory may give more conservative results than that the Fourier's heat conduction theory.
\end{abstract}

Keywords Cracked Substrate, Coating, Hyperbolic heat conduction, Singular integral equations, Crack kinking

\section{Introduction}

High-rate heat transfer has become a major concern in modern industries especially in material processing, such as the pulsed laser heat and ultrasonic waves, and accurate heat conduction analysis is of great importance for the material and structural integrity. Investigation of the temperature and stress fields is essential to the safety design of the composite structures under severe temperature loading. The Fourier heat conduction model, although give sufficient accuracy for many engineering applications, implies infinite thermal wave propagation speed, and renders ineffective at the very small length and time scales associated with small-scale systems [1]. Consideration of the hyperbolic heat conduction model becomes important if irreversible physical processes, such as crack or void initiation in a solid, are involved in the process of heat transport. In these cases, the hyperbolic heat conduction model should be used [2].

Inherent defects in materials such as dislocations and cracks may disturb the temperature distribution when thermal loading is applied, and singularities may be developed in the neighborhood of discontinuities. The singular behavior of temperature gradient around crack tip has been studied based on the classical Fourier heat conduction model [3]. Some investigations on crack problems in thermo-elastic materials have been made using the hyperbolic heat conduction model. The problem of a finite crack in a material layer under transient non-Fourier heat conduction was investigated by Wang and Han [4] and the problem of an interface crack in layered composite media under applied thermal flux was studied using the hyperbolic heat conduction theory [5]. A thermo-elastic analysis of a cracked substrate under a thermal shock was given in Chen and $\mathrm{Hu}$ [6] based on the hyperbolic heat conduction theory; and based on the same theory the transient temperature and thermal stresses around a partially insulated crack in a thermoelastic strip under a temperature impact were obtained [7]. The transient temperature field around a thermally insulated crack in a substrate bonded to a coating has been obtained by using the hyperbolic heat conduction model [8]. 
The thermo-elastic problem of a cracked substrate bonded by a coating under transient thermal loading is studied in this paper using the hyperbolic heat conduction model. Fourier and Laplace transforms are employed to reduce the problem to solving singular integral equations. The asymptotic fields around the crack tip are obtained in an explicit form and Laplace inversion is applied to get the dynamic temperature and stress fields. The crack kinking phenomenon is investigated by applying the criterion of maximum hoop stress.

\section{Statement of the problem and basic equations}

Consider a thermoelastic substrate containing a crack of length $2 c$ parallel to the interface between the substrate and the coating, as shown in Figure 1. The composite is initially at certain temperature and the free surface of the coating $y=-h=-(a+b)$ is suddenly heated to by a temperature change $T_{0}$. The crack surfaces are assumed to be thermally insulated.

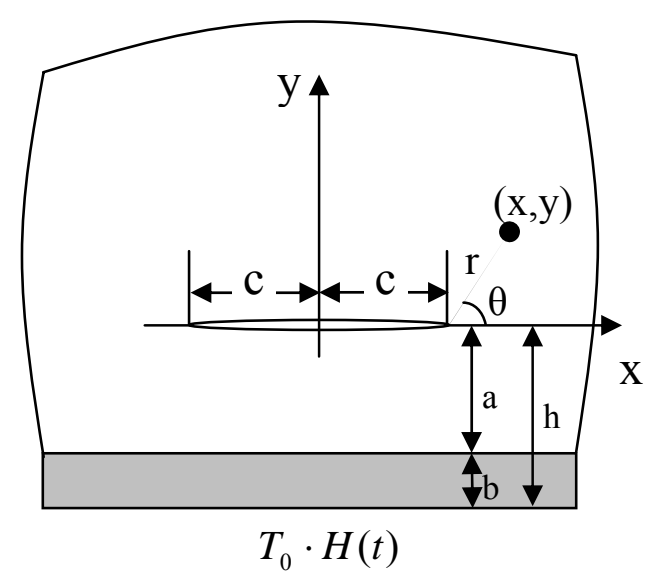

Figure 1. Crack geometry and coordinates.

\subsection{Heat conduction equations}

In the heat-transfer situations include extremely high temperature gradients, extremely large heat fluxes or extremely short transient durations, the Fourier's law may be modified to a relation of the type [9],

$$
q+\tau \frac{\partial q}{\partial t}=-k \cdot \nabla T
$$

where $q$ is the heat flux, $T$ is the temperature, $k$ is the thermal conductivity of the material, $\nabla$ is the spatial gradient operator, and $\tau$ is the so-called relaxation time. The local energy balance equation with vanishing heat source follows:

$$
-\nabla q=\rho C \cdot \frac{\partial T}{\partial t}
$$

where $\rho$ and $C$ are the mass density and the specific heat capacity, respectively. Incorporating Eq. (1) with Eq. (2) leads to the hyperbolic heat conduction equation for the substrate and the 
coating,

$$
d_{i} \cdot \nabla^{2} T^{(i)}=\partial T^{(i)} / \partial t+\tau_{i} \partial^{2} T^{(i)} / \partial t^{2}, \quad(i=1,2)
$$

where $\nabla^{2}$ is Laplace's differential operator, $\tau_{i}, d_{i}=k_{i} /\left(\rho_{i} C_{i}\right)$ and $k_{i}(i=1,2)$ are the relaxation times, the thermal diffusivities and the thermal conductivities of the substrate and the coating, respectively. It is noted that here and afterwards the subscript " $i=1,2$ " denote the quantities of the substrate and the coating, respectively.

By introducing the dimensionless variables

$$
\bar{T}=T / T_{0}, \quad \bar{t}=t d_{0} / c^{2}
$$

where $T_{0}$ is the reference temperature and $d_{0}$ is the reference thermal diffusivity, the governing equations (3) have the following dimensionless forms:

$$
\nabla^{2} T^{(i)}=\frac{\partial T^{(i)}}{\partial t} \frac{d_{0}}{d_{i}}+\frac{\tau_{i} d_{0}^{2}}{c^{2} d_{i}} \frac{\partial^{2} T^{(i)}}{\partial t^{2}}, \quad(i=1,2)
$$

It is noted that here and afterwards, the hat " " " of the dimensionless variables is omitted for simplicity. The hyperbolic heat equation (5) is subjected to the following boundary and initial conditions in dimensionless forms

$$
\begin{gathered}
T(x,-h)=T_{0}, T\left(x,-a^{+}\right)=T\left(x,-a^{-}\right), k_{1} \frac{\partial T\left(x,-a^{+}\right)}{\partial y}=k_{2} \frac{\partial T\left(x,-a^{-}\right)}{\partial y}, \quad(|x|<\infty, t>0) \\
\partial T(x, 0) / \partial y=0,(|x|<c) \\
T\left(x, 0^{+}\right)=T\left(x, 0^{-}\right), \partial T\left(x, 0^{+}\right) / \partial y=\partial T\left(x, 0^{-}\right) / \partial y,(|x| \geq c)
\end{gathered}
$$

It should be noted that the relaxation time $(\tau)$ for most engineering materials are of the order of $10^{-14}-10^{-6} \mathrm{~s}$ and the thermal diffusivity $10^{-8}-10^{-3} \mathrm{~m}^{2} / \mathrm{s}$, and therefore the Fourier parabolic heat conduction model can give good results. Experiments have shown that some special materials may have thermal relaxation time up to the order of $10 \mathrm{~s}$, which are important materials to work as thermal insulators [10]; in this case it is necessary to take into account the effect of the relaxation time and use the hyperbolic heat conduction model. Other applications of hyperbolic heat conduction model are in the area of transient thermal disturbance with small length and time scales.

\subsection{Thermal-elastic field equations}

The equilibrium equations, the strain-displacement relations, the compatibility equation and the constitutive law of plane stress thermoelasticity can be expressed as follows,

$$
\partial \sigma_{x} / \partial x+\partial \sigma_{x y} / \partial y=0, \partial \sigma_{x y} / \partial x+\partial \sigma_{y} / \partial y=0
$$




$$
\begin{gathered}
\varepsilon_{x}=\partial u / \partial x, \quad \varepsilon_{y}=\partial v / \partial y, \quad \varepsilon_{x y}=(\partial u / \partial y+\partial v / \partial x) / 2 \\
\partial^{2} \varepsilon_{x} / \partial y^{2}+\partial^{2} \varepsilon_{y} / \partial x^{2}=2 \partial^{2} \varepsilon_{x y} / \partial x \partial y \\
\varepsilon_{x}=\left(\sigma_{x}-v \sigma_{y}\right) / E+\alpha T, \quad \varepsilon_{y}=\left(\sigma_{y}-v \sigma_{x}\right) / E+\alpha T, \quad \varepsilon_{x y}=(1+v) \sigma_{x y} / E
\end{gathered}
$$

where $E, v$ and $\alpha$ are Young's modulus, Poisson's ratio and the coefficient of linear thermal expansion, respectively.

Let $U(x, y)$ be the Airy stress function, then the stresses can be expresses in terms of $U$ as

$$
\sigma_{x}=\partial^{2} U / \partial y^{2}, \quad \sigma_{y}=\partial^{2} U / \partial x^{2}, \quad \sigma_{x y}=\partial^{2} U / \partial x \partial y
$$

Substitution of Eq. (12) into (10) and (11) leads to

$$
\nabla^{2} \nabla^{2} U+E \cdot \nabla^{2}(\alpha T)=0
$$

By introducing the following dimensionless quantities

$$
\bar{U}=U /\left(E \alpha c^{2} T_{0}\right), \bar{\sigma}_{i j}=\sigma_{i j} /\left(E \alpha T_{0}\right), \bar{\varepsilon}_{i j}=\varepsilon_{i j} /\left(\alpha T_{0}\right),(\bar{u}, \bar{v})=(u, v) /\left(\alpha c T_{0}\right)
$$

the governing equations (13) can be rewritten in the following dimensionless forms:

$$
\nabla^{2} \nabla^{2} U+\nabla^{2}(T)=0
$$

The mechanical boundary conditions can be expressed as

$$
\begin{gathered}
\sigma_{x y}\left(x,-a^{+}\right)=\sigma_{x y}\left(x,-a^{-}\right), \sigma_{y}\left(x,-a^{+}\right)=\sigma_{y}\left(x,-a^{-}\right), \sigma_{x y}(x,-h)=\sigma_{y}(x,-h)=0, \quad(|x|<\infty) \\
\sigma_{x y}(x, 0)=\sigma_{y}(x, 0)=0, \quad(|x|<c) \\
\sigma_{x y}\left(x, 0^{+}\right)=\sigma_{x y}\left(x, 0^{-}\right), \sigma_{y}\left(x, 0^{+}\right)=\sigma_{y}\left(x, 0^{-}\right), u\left(x, 0^{+}\right)=u\left(x, 0^{-}\right), v\left(x, 0^{+}\right)=v\left(x, 0^{-}\right),(|x| \geq c)
\end{gathered}
$$

\section{Temperature field}

Apply Laplace transform to Eqs. (5):

$$
\begin{aligned}
& T^{(i)^{*}}(x, y, p)=L\left(T^{(i)}(x, y, t)\right)=\int_{0}^{\infty} T^{(i)}(x, y, t) \exp (-p t) d t \\
& T^{(i)}(x, y, t)=L^{-1}\left(T^{(i)^{*}}(x, y, p)\right)=\frac{1}{2 \pi i} \int_{B r} T^{(i)^{*}}(x, y, p) \exp (p t) d p
\end{aligned}
$$

where $\mathrm{Br}$ stands for the Bromwich path of integration, and assume the composite is at rest in the beginning, we have:

$$
\nabla^{2} T^{(i)^{*}}=A_{i} p T^{(i)^{*}}+B_{i} p^{2} T^{(i)^{*}}, \quad(i=1,2)
$$

where $A_{i}=d_{0} / d_{i}$ and $B_{i}=\tau_{i} d_{0}^{2} / c^{2} d_{i}(i=1,2)$.

Following the procedure in Chen and $\mathrm{Hu}$ [8], the temperature field in the Laplace domain satisfying the boundary condition and regularity condition can be expressed as 


$$
\begin{gathered}
T^{(1)^{*}}(x, y, p)=\int_{-\infty}^{\infty} E_{1}(\xi) \exp (r y) \exp (-i x \xi) d \xi+W(y), \quad(y \geq 0) \\
T^{(1)^{*}}(x, y, p)=\int_{-\infty}^{\infty}\left[E_{2}(\xi) \exp (r y)+E_{3}(\xi) \exp (-r y)\right] \exp (-i x \xi) d \xi+W(y) \quad(-a \leq y \leq 0) \\
T^{(2)^{*}}(x, y, p)=\int_{-\infty}^{\infty}\left[D_{1}(\xi) \exp (n y)+D_{2}(\xi) \exp (-n y)\right] \exp (-i x \xi) d \xi+V(y) \quad(-h \leq y \leq-a)
\end{gathered}
$$

where $E_{i}(\xi)=E_{i}(\xi, p) \quad(i=1,2,3)$ and $D_{j}(\xi)=D_{j}(\xi, p) \quad(j=1,2)$ are unknowns to be determined, and

$$
\begin{gathered}
W(y)=W(y, p)=f \cdot \exp (-q y), V(y)=V(y, p)=C_{1}^{0} \cdot \exp (m y)+C_{2}^{0} \cdot \exp (-m y) \\
q=\sqrt{A_{1} p+B_{1} p^{2}}, \quad m=\sqrt{A_{2} p+B_{2} p^{2}}, r=\sqrt{\xi^{2}+A_{1} p+B_{1} p^{2}}, \quad n=\sqrt{\xi^{2}+A_{2} p+B_{2} p^{2}}
\end{gathered}
$$

Constants $C_{1}^{0}, C_{2}^{0}$ and $f$ can be obtained from the boundary conditions. As the unknown functions are dependent, all other unknown functions can be expressed by only one independent unknown function, for example $D_{1}(\xi)$, as follows

$$
\begin{gathered}
D_{2}(\xi)=-D_{1}(\xi) / \exp [2 n(a+b)] \\
E_{1}(\xi)=\left[\lambda_{2}(\xi)-\lambda_{1}(\xi)\right] D_{1}(\xi), E_{2}(\xi)=\lambda_{1}(\xi) D_{1}(\xi), E_{3}(\xi)=\lambda_{2}(\xi) D_{1}(\xi) \\
\lambda_{1}(\xi)=\exp (r a)\left(\alpha_{1}+\alpha_{2}\right) / 2, \lambda_{2}(\xi)=\exp (-r h)\left(\alpha_{1}-\alpha_{2}\right) / 2 \\
\alpha_{1}=[1-\exp (-2 n b)] / \exp (n a), \alpha_{2}=n k_{2}[1+\exp (-2 n b)] /\left[r k_{1} \exp (n a)\right]
\end{gathered}
$$

Introduce the temperature density function as

$$
\phi(x)=\phi(x, p)=\partial T^{(1)^{*}}\left(x, 0^{+}, p\right) / \partial x-\partial T^{(1)^{*}}\left(x, 0^{-}, p\right) / \partial x
$$

It is clear from the boundary conditions (27) that

$$
\int_{-c}^{c} \phi(t) d t=0, \quad \phi(x)=0 \quad(|x| \geq c)
$$

Substituting (21) and (22) into (27) and using Fourier inverse transform, we can get

$$
D_{1}(\xi)=\frac{1}{i 4 \pi \lambda_{1} \xi} \int_{-c}^{c} \phi(s) \exp (\text { is } \xi) d s
$$

Substituting (21) and (22) into (7) and applying the relation (29), we get the singular integral equation for $\varphi(t)=\phi(c t)$ as follows

$$
\int_{-1}^{1} \varphi(t)[1 /(t-x)+K(x, t)] d t=2 \pi q f, \quad(|x|<1)
$$

where the kernel function $K(x, t)$ is given as

$$
K(x, t)=\int_{0}^{\infty}[1-R(\xi / c)] \sin [\xi(x-t)] d \xi
$$


and $R(\xi)=r\left(\lambda_{1}-\lambda_{2}\right) / \lambda_{1} \xi$. The singular integral equation (30) under the single-valuedness condition in (28) has the following form of solution [11]:

$$
\varphi(x)=\Psi(x) / \sqrt{1-x^{2}}, \quad|x|<1
$$

where $\Psi(x)$ is a bounded and continuous function. From the condition (28), it is clear that $\Psi(x)$ is an odd function of $x$, i.e., $\Psi(-x)=-\Psi(x)$.

Function $\Psi(x)$ can be solved numerically, as detailed in $\mathrm{Chen}$ and $\mathrm{Hu}$ [8], and function $D_{1}(\xi)$ can be calculated by using the Chebyshev quadrature for integration as

$$
\begin{gathered}
D_{1}(\xi) \cong \frac{c}{4 \pi \xi \lambda_{1}} \sum_{i=1}^{n} w_{i} \Psi\left(x_{i}\right) \sin \left(c \xi x_{i}\right) \\
w_{i}=\pi / n, \quad x_{i}=\cos [(2 i-1) \pi / 2 n], \quad i=1,2, \ldots, n
\end{gathered}
$$

By substituting Eq. (33) into (21)-(23) the temperature in the Laplace domain can be obtained. The temperature in the time domain can be determined by applying the Laplace inverse transform.

\section{Thermal stress field}

Considering the temperature expressions (21-23), the general solution of the Airy function satisfying the regular condition at infinity can be expressed as

$$
\begin{array}{rlr}
U^{*}= & \int_{-\infty}^{\infty}\left[\left(A_{1}+A_{2} y\right) \exp (|\xi| y)+G_{11}(\xi) \exp (-r y)\right] \exp (-i x \xi) d \xi-W(y, p) / q^{2} \quad(y \geq 0) \\
U^{*}= & \int_{-\infty}^{\infty}\left[\left(B_{1}+B_{2} y\right) \exp (|\xi| y)+\left(B_{3}+B_{4} y\right) \exp (-|\xi| y)\right] \exp (-i x \xi) d \xi & (-a \leq y \leq \\
& +\int_{-\infty}^{\infty}\left[G_{21}(\xi) \exp (r y)+G_{22}(\xi) \exp (-r y)\right] \exp (-i x \xi) d \xi-W(y, p) / q^{2} \quad \\
U^{*}= & \int_{-\infty}^{\infty}\left[\left(C_{1}+C_{2} y\right) \exp (|\xi| y)+\left(C_{3}+C_{4} y\right) \exp (-|\xi| y)\right] \exp (-i x \xi) d \xi & (-h \leq y \\
& +\int_{-\infty}^{\infty}\left[G_{31}(\xi) \exp (m y)+G_{32}(\xi) \exp (-m y)\right] \exp (-i x \xi) d \xi-V(y, p) / m^{2} \quad
\end{array}
$$

where $B_{i}, C_{i}(i=1-4), A_{1}$ and $A_{2}$ are unknowns to be determined, and $G_{i j}(\xi)$ $(i=1-3, j=1,2)$ are known functions determined from the boundary conditions.

Denote the jumps of displacement across the plane $y=0$ by $\langle u\rangle$ and $\langle v\rangle$,

$$
\langle u\rangle=u\left(x, 0^{+}, p\right)-u\left(x, 0^{-}, p\right),\langle v\rangle=v\left(x, 0^{+}, p\right)-v\left(x, 0^{-}, p\right)
$$

Following the procedure in Jin and Noda [12] and introducing two dislocation density functions $f_{j}(x) \quad(j=1,2)$ as 


$$
f_{1}(x)=f_{1}(x, p)=\partial\langle u\rangle / \partial x, f_{2}(x)=f_{2}(x, p)=\partial\langle v\rangle / \partial x
$$

By applying the boundary conditions (16-18), it can be shown that $f_{i}(x)(i=1,2)$ satisfy the following singular integral equations:

$$
\begin{aligned}
& \int_{-c}^{c} f_{1}(t)\left[1 /(x-t)+M_{11}(x, t)\right] d t+\int_{-1}^{1} f_{2}(t) M_{12}(x, t) d t=U_{1}(x) \\
& \int_{-c}^{c} f_{1}(t) M_{21}(x, t) d t+\int_{-1}^{1} f_{2}(t)\left[1 /(x-t)+M_{22}(x, t)\right] d t=U_{2}(x)
\end{aligned}
$$

where functions $U_{i}(x), M_{i j}(x, t)(i, j=1,2)$ are known functions. Functions $f_{j}(x) \quad(j=1,2)$ also need to satisfy the single-valuedness conditions

$$
\int_{-c}^{c} f_{j}(x) d x=0, \quad(j=1,2)
$$

The solution of the integral Eqs. (39), $f_{j}(x) \quad(j=1,2)$, can be expressed as follows

$$
f_{j}(c t)=F_{j}(t, p) / \sqrt{1-t^{2}}, \quad(j=1,2)
$$

and the singular integral equations can be reduced to solving a system of algebraic equations in terms of $F_{1}(t, p)$ and $F_{2}(t, p)$, see $[6,7]$.

Following the procedure in Chen and $\mathrm{Hu}[9]$, the stress intensity factors (SIFs) $K_{1}^{*}(p)$ and $K_{2}^{*}(p)$ are obtained as

$$
K_{1}^{*}(p)=-\sqrt{\pi c} F_{2}(1, p) / 4, K_{2}^{*}(p)=-\sqrt{\pi c} F_{1}(1, p) / 4
$$

and the dynamic singular stresses near the crack tip are

$$
\begin{gathered}
\sigma_{y}(r, \theta, t)=\sqrt{\frac{c}{2 \pi r}}\left\{K_{1}(t) \cos \left(\frac{\theta}{2}\right)\left[1+\sin \left(\frac{\theta}{2}\right) \sin \left(\frac{3 \theta}{2}\right)\right]+K_{2}(t) \sin \left(\frac{\theta}{2}\right) \cos \left(\frac{\theta}{2}\right) \cos \left(\frac{3 \theta}{2}\right)\right\} \\
\sigma_{x y}(r, \theta, t)=\sqrt{\frac{c}{2 \pi r}}\left\{K_{1}(t) \sin \left(\frac{\theta}{2}\right) \cos \left(\frac{\theta}{2}\right) \cos \left(\frac{3 \theta}{2}\right)+K_{2}(t) \cos \left(\frac{\theta}{2}\right)\left[1-\sin \left(\frac{\theta}{2}\right) \sin \left(\frac{3 \theta}{2}\right)\right]\right\} \\
\sigma_{x}(r, \theta, t)=\sqrt{\frac{c}{2 \pi r}}\left\{K_{1}(t) \cos \left(\frac{\theta}{2}\right)\left[1-\sin \left(\frac{\theta}{2}\right) \sin \left(\frac{3 \theta}{2}\right)\right]-K_{2}(t) \sin \left(\frac{\theta}{2}\right)\left[2+\cos \left(\frac{\theta}{2}\right) \cos \left(\frac{3 \theta}{2}\right)\right]\right\}
\end{gathered}
$$

where the dynamic SIFs $K_{1}(t)$ and $K_{2}(t)$ are given by

$$
K_{1}(t)=L^{-1}\left(K_{1}^{*}(p)\right), K_{2}(t)=L^{-1}\left(K_{2}^{*}(p)\right)
$$

and $(r, \theta)$ are the polar coordinates measured from the crack tip defined by 


$$
r^{2}=(x-c)^{2}+y^{2}, \quad \tan (\theta)=y /(x-c)
$$

The hoop and shear stresses at an angle $\theta$ near the crack tip can be obtained in terms of the polar coordinates $(r, \theta)$ as

$$
\begin{aligned}
& \sigma_{\theta \theta}(r, \theta)=\sigma_{y}(r, \theta) \cos ^{2} \theta+\sigma_{x}(r, \theta) \sin ^{2} \theta-\sigma_{x y}(r, \theta) \sin 2 \theta \\
& \sigma_{r \theta}(r, \theta)=\sin 2 \theta\left[\sigma_{y}(r, \theta)-\sigma_{x}(r, \theta)\right] / 2+\sigma_{x y}(r, \theta) \cos 2 \theta
\end{aligned}
$$

The hoop stress intensity factor and shear stress intensity factor associated with the hoop and shear stresses at an arbitrary angle $\theta$ can be defined as:

$$
K_{\theta \theta}=\lim _{r \rightarrow 0}\left(\sqrt{2 r} \sigma_{\theta \theta}\right), K_{r \theta}=\lim _{r \rightarrow 0}\left(\sqrt{2 r} \sigma_{r \theta}\right)
$$

\section{Numerical results and discussion}

The effect of the thickness of coating $b / c$ on the dynamic SIFs is shown in Figure 2 when the geometry of the cracked substrate is $a / c=1$, the material properties are $k_{1}=k_{2}=1, v=0.4$ and $\tau_{1}=1, \tau_{2}=0.5$ and the boundary temperature condition $T_{0}=+1$. The SIFs oscillate and increase in magnitude till they reach the peak values and oscillate for some time before approaching their static values. The magnitude of the peak value decreases as the size ratio $b / c$ increases, which indicates that a thicker coating may decrease the magnitude of SIF.

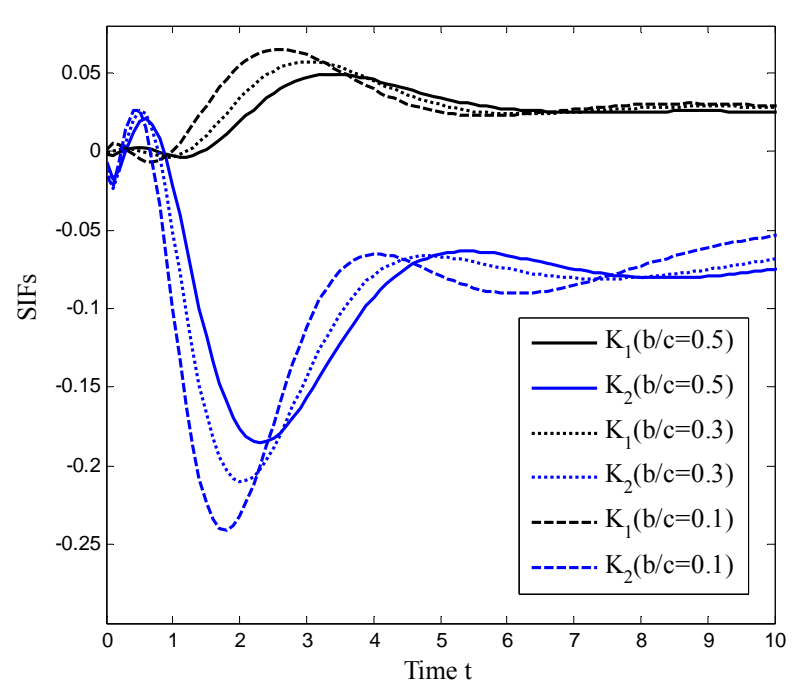

Figure 2. Effect of $b / c$ on the dynamic SIFs when $a / c=1$.

Figure 3 exhibits the variation of the dynamic SIFs with different thermal relaxation times $\tau_{1}$ and $\tau_{2}$ when $k_{1}=k_{2}=1, v=0.4, a / c=1, b / c=0.5$ and $T_{0}=+1$. The results based on the Fourier heat conduction is the special case when $\tau_{1}=\tau_{2}=0$, which shows that the dynamic SIF increases smoothly to reach the peak values and then decrease to the static values. The oscillating feature of the dynamic SIFs is observed when the hyperbolic heat conduction theory is applied, and as the relaxation time of the coating decreases the magnitude of the dynamic SIF decreases. This conclusion indicates that the structure safety design based on the hyperbolic heat conduction theory may lead to more conservative design than Fourier's heat conduction theory. 


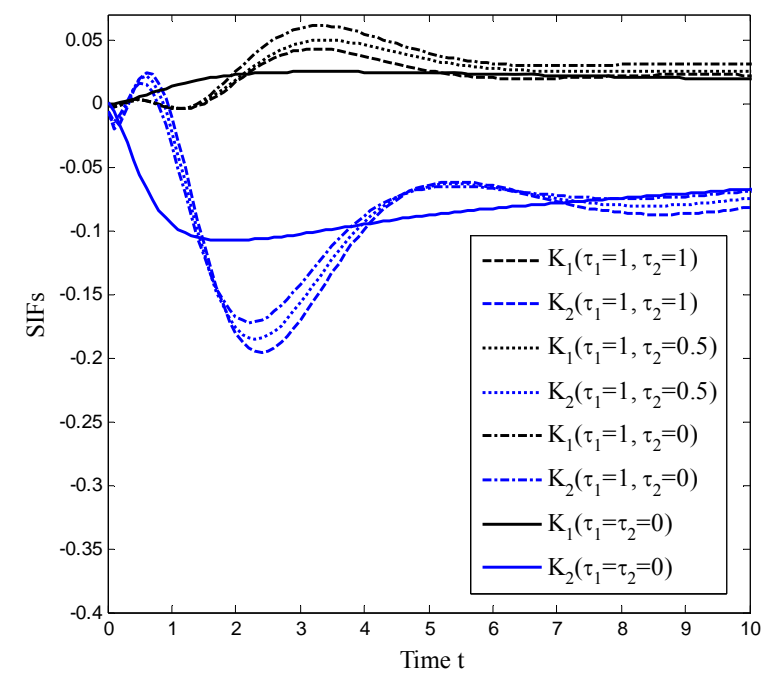

Figure 3. Effect of relaxation time on dynamic SIFs.

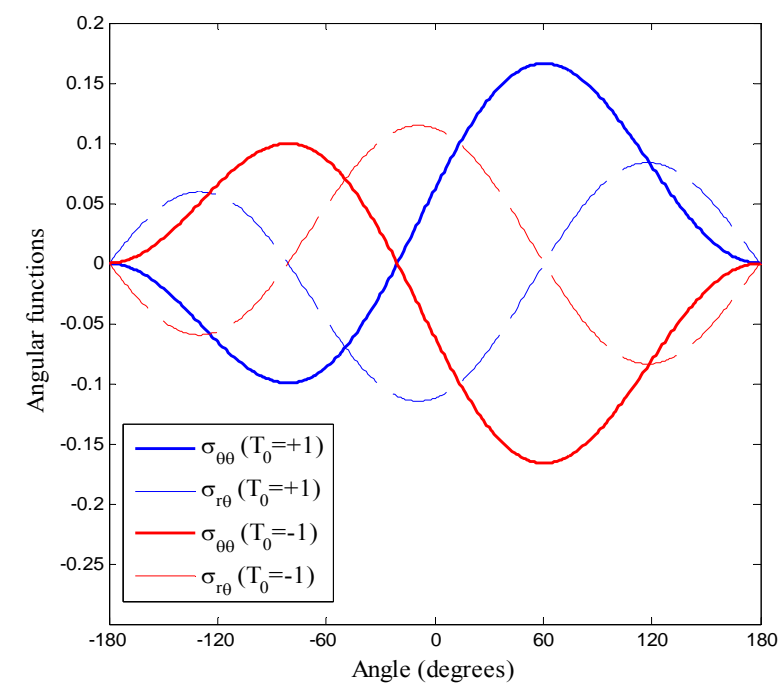

Figure 4. Angular functions of the normalized hoop stresses and shear stresses at a time instant.

The angular functions of the normalized dynamic hoop stresses $\sigma_{\theta \theta} \sqrt{2 \pi r / c}$ and shear stresses $\sigma_{r \theta} \sqrt{2 \pi r / c}$ at a specific time instant $t=3.0$ are displayed in Figure 4 when $a / c=1, b / c=0.1$, $k_{1}=k_{2}=1$ and $v=0.4$. The maximum hoop stress criterion is used to study crack kinking in this paper. It is shown that when the hoop stress reaches the maximum value, the shear stress is zero. When the temperature loading on the surface of the coating is positive, the maximum hoop stress appears at the angle $\theta=+60$ degrees, which indicates that the crack may kink in this direction; when a negative temperature is applied on the coating surface, the hoop stress reaches the maximum value at the angle $\theta=-70$ degrees, which means that the crack may kink toward the coating in this direction. It seems that high temperature loading on the surface may lead to crack kinking away 
from the surface and low temperature loading may cause the crack to kink toward the coating.

\section{Conclusions}

The transient thermal stresses around a crack in a substrate bonded to a coating have been obtained using the hyperbolic heat conduction theory. Integral transform method is applied and the hyperbolic heat conduction and thermo-elastic crack problem are reduced to solving singular integral equations. The crack kinking phenomenon is investigated by applying the criterion of maximum hoop stress. Numerical results show that the hyperbolic heat conduction parameters, the material properties and the geometric size of the composite have significant influence on the dynamic stress field. Hot or cold temperature loadings may lead to different crack kinking directions. The results predicted by the hyperbolic heat conduction model are more conservative than that by the Fourier's heat conduction model.

\section{References}

[1] M.H. Babaei, Z.T. Chen, Transient hyperbolic heat conduction in a functionally graded hollow cylinder. J Thermophys Heat Transfer, 24 (2010) 325-330.

[2] M. Naji, M. Al-Nimr, T. Darabseh, Thermal stress investigation in unidirectional composites under the hyperbolic energy model. Int J Solids Struct, 44 (2007) 5111-5121.

[3] D.Y. Tzou, Characteristics of thermal and flow behavior in the vicinity of discontinuities. Int $\mathbf{J}$ Heat Mass Transfer, 35 (1992): 481-491.

[4] B.L. Wang, J.C. Han, A crack in a finite medium under transient non-Fourier heat conduction. Int J Heat Mass Transfer, 55 (2012) 4631-4637.

[5] B.L. Wang, J.C. Han, Non-Fourier heat conduction in layered composite materials with an interface crack. Int J Eng Sci, 55 (2012) 66-75.

[6] Z.T. Chen, K.Q. Hu, Thermo-elastic analysis of a cracked half-plane under a thermal shock impact using the hyperbolic heat conduction theory. J Therm Stresses, 35 (2012) 342-362.

[7] K.Q. Hu, Z.T. Chen, Thermoelastic analysis of a partially insulated crack in a strip under thermal impact loading using the hyperbolic heat conduction theory. Int J Eng Sci, 51 (2012) 144-160.

[8] Z.T. Chen, K.Q. Hu, Hyperbolic heat conduction in a cracked thermoelastic half-plane bonded to a coating. Int J Thermophys, 33 (2012) 895-912.

[9] J.D. Achenbach, Wave propagation in elastic solids, North-Holland, 1973.

[10]Y.M. Ali, L.C. Zhang, Relativistic heat conduction. Int J Heat Mass Transfer, 48 (2005) 2397-2406.

[11]F. Erdogan, Complex function technique, in: A.C. Eringen (Editor), Continuum Physics. Vol. 2, Academic Press, New York, 1975, pp. 523-603.

[12]Z.-H. Jin, N. Noda, An internal crack parallel to the boundary of a nonhomogeneous half plane under thermal loading. Int J Eng Sci, 31 (1993) 793-806. 